\title{
Coping with Complexity
}

\author{
MANFRED KOCHEN
}

\author{
University of Michigan, USA
}

(Received May 1979)

\begin{abstract}
We offer a new typology of problems according to such attributes as the fuzzy set of possible solutions, the fuzzy set of properties a solution should have, the fuzzy set of knowledge necessary and sufficient to find a solution, deadlines and constraints. Problems are ranked by degree of 'structuredness' from well-structured to ill-defined. Some real problems are shown to be ill-defined and the causes of their inherent imprecision are traced. A model for coping with ill-defined problems enables us to estimate an optimal degree of precision in both dealing with the problem and in requesting information to help cope with it. This has interesting implications for the design of information systems.
\end{abstract}

\section{INTRODUCTION}

WHEN SimON [15] completed his study of administrative behavior, he had shifted the emphasis from the art of 'getting things done' that is, from the study of action, to the study of decision-making or the determination of what is to be done. The 8-yr period of Simon's study was also one of exceptional creativity in the development of a mathematical foundation for decision theory $[18,19]$. A decision problem was regarded to be specified by (1) a set of alternative acts, (2) a set of possible states that the decision-maker's world could be in after an action is to be taken and (3) the net benefit that the decision-maker can expect from each stateaction contingency.

We call 'problem solving' the activity of making a rational choice among the options when that requires us to use expert knowledge and lengthy chains of deductions or inductive inferences. Playing chess differs from gambling in a lottery in that we need to think and know more in deciding on a move than we do in placing a bet. It is therefore more typical of problem-solving than, for example, poker. 'Rational' does not necessarily refer to adherence of criteria for optimization or maximization of expected utility but to the behavior of people in risky choice situations according to laws and psychologically significant criteria that we are only now beginning to discover [17].

Amarel $[1,2]$ has conceptualized a 'problem' as being a statement that specifies three sets:

(1) The set of all possible solutions, illustrated by the italicized phrase in "Find all integer pairs $(x, y)$ such that $3 x+17 y=51$ using Euclid's algorithm."

(2) The set of properties a solution should have, illustrated by the phrase " $3 x+17 y=51 "$ in the above problemstatement.

(3) The set of statements representing what is known that is necessary and sufficient to find a solution, illustrated by the phrase "using Euclid's algorithm" in the above problem-statement.

Computers have been tools for vastly amplifying our ability to solve problems that are reducible to arithmetic. But they are now also recognized as tools for transforming our very conception of problems and problem-solving. That has a far greater impact. Recent models of human information processing have led to new approaches to the study of human problemsolving $[5-7,10,12]$. Higher-level program- 
ming languages have enabled a computer user merely to express his problem-not necessarily a mathematical one-in that lizinguage with the expectation that the computer system (compiler, etc.) will work out the detailed steps of a solution procedure as well as their execution.

It seems that most of our education, from kindergarten to graduate school, trains us for problem-solving in this sense. Yet, it is ironic that most of us seldom encounter situations outside schools, laboratories or computer systems that necessitate the making of decisions that we recognize as resembling the so-called 'problems' to which we refer in the above sense. Our most important choice tasks, such as choosing a job or a career are not 'problems' in this sense. Those who are highly skilled in such problem-solving (even with the aid of sophisticated computers) are often not the best equipped to do well in real choice situations. According to a more realistic model of problem solving [11], this process involves: (1) conceptualization; (2) modeling; (3) model-solving; (4) implementation, as systemically integrated subprocesses. The corresponding attempt to analyże this model, however, still assumes the existence of a well-defined time-varying goal $G(t)$ and a performance function $a(t)$ such that discrepancies between the two can be specified and lessened. It is doubtful that the mathematical representation used in such models, e.g. conceptualizing $G$ and $a$, so that $G-a$ (subtraction) is meaningful, captures the essence of 'problem-solving' in real life. We propose in this paper to reexamine our conceptualization of what a 'problem' is with specific emphasis given to the 'ill-structured' problem. Specifcally, we replace the notion of a 'problem' by that of a 'task-situation'. We propose to replace 'problem-solving' by the term 'coping'. We argue that we are likely to cope well with simple task-situations when our representation of these situations is as precise as possible. However, if a task-situation is complex, we fail to cope if we represent the situation not only too vaguely but similarly if we view it with excessive precision. We find, in fact, that there is an optimal degree of precision with which to view complex task-situations.

The new idea we propose here is to characterize a problem not only by (1) the set of all possible solutions, (2) the set of properties a solution should have and (3) the knowledge necessary and sufficient to find a solution, but on the basis of criteria based on constraints of (4) time, (5) information processing and (6) emotional and social factors as well. The importance of time constraints or deadlines is of course well known to every manager, whether he manages only himself or others. A 'solution' after the deadline is no solution. Even in schools and psychological laboratories there are implicit time constraints, though neither teachers/experimenters nor students/subjects always regard these explicitly as part of the task-situation. Information-processing constraints are another important part of a tasksituation that should be explicitly recognized. (Simon was among the first to stress this). A shopper in a supermarket may be aware that he/she could buy a 'better' basket of commodities (e.g. meeting nutritional requirements, least cost) with the help of a linear program than by browsing and responding to advertisers' cues etc., but unless he or she had a programmed calculator to do this (with all necessary coefficients-e.g. the number of grams of protein $/ \mathrm{gm}$ for each item of interest in the supermarket), the necessary information processing capacity is lacking.

The social and emotional constraints in the task-situation have, to our knowledge, not yet been identified. Possibly they may have the greatest importance among the six attributes of a task-situation. Even if the supermarket shopper had the required information processing capacity, he or she may be unwilling to be seen by acquaintances as someone who computes what to buy. The computation could be made at home, with a catalog as input to the home computer and a shopping list or order as output, and the order could even be automatically placed, filled and processed. But the shopper might miss browsing in the supermarket not only among the goods but amongst the other shoppers as well.

There is a second new idea of great importance that we now introduce. That is the precision of these six sets of attributes of a tasksituation. The set of possible choices between $a$ and $b$ is less precise than the set of choices among values of a probability with which to make one of the two choices. It is less precise because it does not specify whether the choice between $a$ or $b$ is to be made by tossing a coin, 
casting a die, according to a pattern, rule, etc. Minimizing the expected loss is a more precise statement of a property desired in a solution than 'winning as often as possible'.

\section{SIMPLE TASK SITUATIONS}

It is plausible that for such tasks as an oddseven game, (a simple task situation in which each of two players can simultaneously put forth one or two fingers with one player winning if the combined number of fingers is even) the greater the precision in specifying (1) the set of possible solutions, (2) the desired properties of a solution and, (3) the knowledge needed to find a solution, the greater the chances of finding a satisfactory solution. This is not evident for the attributes 4-6 mentioned in the previous section. Specifying with great precision a deadline by which a choice in the game must be made may be less productive than leaving some latitude for loose interpretation; total vagueness is counter-productive as well because it confuses.

Similarly, too precise a specification of information processing requirements could incur the risk of overestimation resulting in unused capacity or inefficient use of costly resources or of underestimation, leading to ineffectiveness or other inefficiencies. Too little precision in specifying needed information processing capacity gives no guidance with respect to the means by which the needed resources may be procured. There may be an optimal degree of precision providing the decision-maker with the flexibility to adapt to the needs as he learns them. Finally, very precisely specified criteria for affect or social interaction engender excessive rigidity that makes behavior appear automatic and inhuman, while totally unspecified criteria lead to permissiveness and uncontrolled behavior. Both extremes are generally counterproductive.

As an example of a counterproductive way of representing a simple-task situation, let us take the case of two individuals engaged in a game in which each can simultaneously put forth one or two fingers. One player receives a penny from the other if the combined number of fingers is odd and vice-versa if it is even. This can be accurately represented as a zerosum game with payoff matrix

$$
\begin{aligned}
& 1 \text { finger } \\
& 2 \text { fingers }
\end{aligned}\left[\begin{array}{rr}
1 & -1 \\
-1 & 1
\end{array}\right]
$$

for one of the players and $\left(\begin{array}{rr}-1 & 1 \\ 1 & -1\end{array}\right)$ for his opponent.

A poor representation of this situation is one in which a person believes that, at each move: (The parentheses denote a set)

(1) his options for action are: ('one finger', 'two fingers');

(2) the basis for his choice should be to: (win as often as possible, beat the opponent);

(3) what he needs to know to decide is: (a pattern in the opponent's choices, a telepathic signal);

(4) his time constraints are: (to decide in less than a second or so);

(5) his information processing requirements are: (to consider only elementary patterns, to guess without thinking);

(6) his psycho-social criteria are: (to appear as a regular person rather than one who calculates his moves, to conceal his decision by maintaining a pokerface).

Most people who play this do not know of or think of the idea of a mixed strategy in a zerosum game. Yet, it can be experimentally verified that they select one finger at random approximately half the time, just as game theory predicts. It is a very simple task to perform, partly because it does not depend greatly on the quality of how a player represents the task to himself.

While, for attributes (1)--(3) of a simple tasksituation, coping appears to be enhanced by the maximum precision that the decisionmaker is capable of, there may be an optimum degree somewhere between the lowest and highest degree of precision in specifying attributes (4)-(6). The simpler the task-situations, the closer that optimum degree is to the maximum.

\section{TOWARD GREATER COMPLEXITY}

What makes a task-situation more complex? We extend the definition of complexity used in 
computer science so that not only task-situations with greater information-processing requirements but also those which cannot be readily (or at all) represented as well-defined problem-statements are regarded as more complex. As an example of a task-situation of intermediate complexity, consider a person in a moderately satisfying job who is offered a new job with much better pay. One representation of six attributes of this problem-situation is:

(1) Options $=($ Keep present job, accept new job).

(2) Desiderata of choice $=$ (Maximize income, optimize self-actualization).

(3) Needed knowledge $=$ (Detailed data regarding new job, awareness of priority feelings).

(4) Time criterion $=$ (Decision in two weeks, decision in two months).

(5) Information Processing Criteria $=$ (Analytic reasoning, 'Clinical' judgment).

(6) Psychic Criteria $=(\mathrm{Be}$ and appear decisive, rigidity).

In terms of the self-actualization criteria, the decision-maker could represent attributes (1) and (2) in the form of a payoff matrix, as follows:

\begin{tabular}{lcc} 
& $\begin{array}{c}\text { Keep } \\
\text { present job }\end{array}$ & $\begin{array}{c}\text { Take } \\
\text { new job }\end{array}$ \\
\cline { 2 - 3 } $\begin{array}{l}\text { Feeling of high self- } \\
\text { actualization, incl. pay }\end{array}$ & $\begin{array}{c}\text { Net benefit } \\
\text { High }\end{array}$ & $\begin{array}{c}\text { Net benefit } \\
\text { Very high }\end{array}$ \\
\hline $\begin{array}{l}\text { Feeling of low or med. } \\
\text { self-actualization, incl. pay }\end{array}$ & $\begin{array}{c}\text { Net benefit } \\
\text { Very low }\end{array}$ & $\begin{array}{c}\text { Net benefit } \\
\text { low }\end{array}$ \\
\hline
\end{tabular}

$\mathrm{He}$ is uncertain about his future state of wellbeing, especially if he takes the new job. There are at least two kinds of uncertainty. One is illustrated with the example of a person's inability to predict whether a tossed coin will land heads or tails; another, by whether a tossed coin is 'true' or 'false'. The categorization of coins into 'true' vs 'false' is a particular representation. It reflects a particular state of (imperfect) understanding (as opposed to confusion), much as does a classification of job- satisfaction criteria into income and self-actualization criteria. The further classification of self-actualization into high and low degrees illustrates yet another degree of constrained freedom in the hierarchical representation of a task-situation. The decision-maker is free to categorize self-actualization in many ways but the choices are subject to a constraint in so far as they all must pertain to self-actualization.

Kahneman and Tversky [17] showed that preference among two hypothetical job offers depends on the subject's present position. If he is earning $\$ 17,000$ with a $30 \%$ chance of promotion within three years, he is $1 \frac{1}{2}$ times as likely to prefer a change to $(\$ 18,000$ annual salary, $60 \%$ chance of promotion in 3 years over a change to $(\$ 16,500,90 \%)$. If, on the other hand, his present position is $(\$ 15,000$, $75 \%$ ), then he is 8 times as likely to prefer a change to $(\$ 16,500,75 \%)$ over a change to $(\$ 18,000,60 \%)$.

Different decision-makers differ with respect to how they understand, imagine, conceptualize, a task-situation. This is reflected in their representation. That preferences vary with a decision-maker's frame of reference is well known [17]. Different laws appear to govern the behavior of people in dealing with uncertain and imprecise situations than the laws of rational behavior according to probabilistic decision theory-e.g. maximizing expected value.

Clearly, a precise representation of all aspects of a completely random system is impossible. But while certain macroscopic attributes can be described with precision, all microscopic details cannot. This imposes an implicit limitation on the facility with which an individual can cope with a complex (i.e. uncertain or random) task-situation.

It would appear that randomness is not the only aspect of a task-situation that makes it complex. The cardinality and fullness of the sets used in specifying the six attributes also contribute importantly to complexity. The set of possible solutions for a complex situation involves more variables and a larger range for each variable than is the case for a simpler situation. For example, the number of firstorder variables considered in the design of cars in the 1970's was greater than the number in the 1950s when pollution, for instance, was only a second-order effect in light of fewer existing cars. 
The set of properties a solution should satisfy is larger for a more complex situation. Some of the desiderata that are added as complexity increases originate from greater interrelations among variables. For example, relations among variables in designing cars in the 1970 s still need take but little account of the trends in communication. As both transportation and communication technologies develop, the demand patterns for each may begin to interact. The interactions add additional properties and constraints that desired 'solutions' should satisfy.

The amount of relevant knowledge useful in finding a solution is much greater - as well as more difficult to specify, find, understand and use - as the task-situation increases in complexity. Stakes are higher and more irreversible. Complex task-situations demand more of a break with traditional ways of thinking than do simple ones. A task that is but a minor variant of one we encounter frequently is not complex. A task that is quite unlike any we have ever encountered is complex because we must think quite differently about it. More massive shifts in representation, in our conceptualization, are required. Relevant knowledge is far more difficult to identify and recognize. Time constraints are more demanding for complex tasks, and information processing requirements are greater. In this respect, a computationally complex task-situation is also complex in our sense. More and more subtle social and psychological criteria also increase complexity.

\section{REPRESENTATIONS OF COMPLEX TASK-SITUATIONS}

Among the most complex task-situations are those involving human systems, particularly those in which many diverse people interact in ways that give rise to new forms of social organization, and to new self-generating behavior patterns. We can imagine, for example, a small group of people in a community having to decide on a new mayor or chief of some service. Suppose that they agree to meet in order to select the candidates, in essence, the set of possible 'solutions'. Each participant may bring with him a different agenda. The first task is to reach some agreement about the agenda, about a consensual way of viewing the common task- situation. If each participant starts with a slightly different understanding, then a common image of the situation may emerge as the members learn-i.e. revise and adjust their images by mutal interaction.

The task-situation includes the participants. If they start with very diverse images or points of view, then the situation is more complex. and it may take far longer for them to reach a common understanding if they reach one at all. If one participant's outlook is primarily political, with a cognitive style characterized by concreteness and action-orientation (rather than reflectiveness) while another is primarily rational, abstract and reflective, a meeting of minds may be hard to reach.

Agreement on what properties a solution should have is harder to reach if the participants have different value systems. Only after they have converged on at least an approximation of a common image of the task-situation can they proceed to resolve their value conflicts. This is obviously more difficult when values are far apart, and thus task-situations which involve such diverse participants tend to be more complex. The most acrimonious controversies can arise over the qualifications of the ideal candidate for the post. Often political, rational and normative aspects are confused and interchanged in the debate, with a participant arguing for the importance of some trait that his favorite candidate happens to have. The reasons, however, why he may favor that candidate may focus primarily on political self interest (who controls what).

After many participants have managed to share some perception of the task situation by agreeing on the options and even on the desiderata of an ideal option, it might be assumed that the knowledge needed to choose the candidate could be easily identified, found and used, because there is a greater chance for at least one of the participants to have the required expertise or experience. However, if the diversity of expertise is fragmented and uncoordinated, the ensuing search can just as easily turn into a cacaphonous Babel. People who come from vastly different intellectual worlds do not speak the same language. The cycle of attempting to share conceptualizations, points of view, images and knowledge, followed by attempts to resolve value conflict merely starts over, but at a different level. 
Different participants in a situation also have diverse perspectives about time. Some understand the art of strategically timing key decisions, while others are oblivious to the passage and value of time. A task-situation in which the timing of group decisions to be made is ambiguous or uncertain, possibly due to widely differing views about when the decision is due, is complex if only because the participants may expand their energies and budget their time in totally incompatible ways, leading to interference, divergence, confusion and fragmentation. One of the key tasks of a coordinator is to synchronize the time schedules of various participants.

Does the information processing capacity of a group of information processors exceed that of the individuals in a super-additive way? A conjecture from studies on the logic of induction suggests that it can. An expanding, interacting community of interactive trial and error machines will eventually identify any partial recursive function, with each member of the community receiving a non-terminating sequence of ordered pairs that constitute the function [14]. Thus, an ever-growing community of machines, each of which can eventually identify the relations of the form "There exists $x_{1}$ such that $R^{2}\left(x_{1}, x\right)$ " for all two-place relations $R^{2}$ can eventually identify relations of the form "There exists $x_{1}$ and for all $x_{2}$ such that $R^{3}\left(x_{1}, x_{2}, x\right)$ " for all three-place predicates $R^{3}\left(\Sigma_{2}\right.$ of the Kleene Arithmetic Hierarchy). In this sense, an expanding community can generate (as well as cope with) more complex situations than a single member.

Finally, the affective and social aspects of a situation often become very pronounced only when there are many participants in a situation. If diversity of cognitive styles contributes to situational complexity, then the diversity of emotional response patterns and social interaction styles complicates it further. A major source of this complexity is a lack of awareness not only regarding how others feel and how they are affected by our behavior, but by our own feelings and likely reactions directed at the behavior of others. Another source of complexity are the social traps [13] or 'catch-22' situations that are often generated by certain people, especially when they gravitate toward others like themselves in an effort to create selfperpetuating 'catch-22' situations. A person may, for example, nominate one of his peers to an open post only then to present all the reasons why he would not be a good choice, or get the candidate elected and then do everything he can to keep him from controlling the situation. Situations characterized by social complexity present participants with difficult trade-offs between seemingly conflicting options, options of which they are frequently unaware or by which they feel entrapped. It is this latter feeling that contributes to the fundamental complexity of task-situations.

\section{COPING BY MODIFYING REPRESENTATIONS}

Living systems, and human systems, in particular, have a remarkable ability to survive, to 'muddle through' task-situations which our most cleverly designed and most 'intelligent' non-living systems would very probably not survive. If the rule 'that anything that could go wrong will go wrong' applies to the complex systems we build, then a rule such as 'anything that could go right will go right' may apply to the human systems that have naturally evolved. Deliberate efforts to destroy a species or a defunct human institution have often proved to be quite unsuccessful, as these systems, like new strains of bacteria or weeds, develop unforseeable forms of resistance and resilience faster than they can be overcome. The concept of coping applies to adaptive survival in human systems that we value; it also applies to bacteria that we value negatively (e.g. mutants of viruses in hospitals that adapted to resist antibiotics). We confine our attention here to its use in human systems in which how the participants represent their task-situation is the primary aspect of coping.

\section{PRECISION AND ITS EFFECT ON THE REPRESENTATION}

We focus even more sharply on one aspect of a representation: its precision. We now try to show that for a complex task-situation, an ideal representation has an intermediate rather than an extreme degree of precision. As an example, we analyze the task-situation in which one member of a department is to be chosen as its chairman and persuaded to accept. Snow [16] has provided a masterful de- 
scription (fictional) of how a college in Cambridge coped with such a situation.

Suppose that only two ways of imagining the situation exist among the group members: (1) only people who do not threaten the vital interest of key people in the group are acceptable; (2) anyone with a sufficiently strong personality and capable of leadership is acceptable. Adherents of neither view are or care to be too precise in specifying 'vital interests', 'strength of personality', etc. Yet each sufficiently understands the others' interpretation of these terms so that he feels concerned and objects. One participant who feels he ought to be chairman may try to persuade others that he should be a candidate, and he may in the process make different, and even contradictory statements to different potential constituents. The truth of these statements may be secondary to their effectiveness in persuading listeners to vote for him. Astute listeners are aware of this, and also of the possibility that the rare candidate who speaks only the truth may not be very promising. How can they cope with the first task, of setting up a set of possible solutions, i.e. of candidates? If they imagine some ideal candidate in too much detail and too exactly, no one will fit; nor will they agree. If they imagine him too vaguely, they have made no progress. Successful professional imagebuilders often select just the right degree of precision.

Coping with value conflicts can also be helped by the deliberate attempt to avoid both excess precision and excess vagueness. Timehonored statutes and moral maxims reflect these compromises in their language. 'Thou shall not kill' owes its force partly to its lack of specificity. The potency would be diminished if qualified by numerous clauses such as 'except in the case of war', or 'any normal human being after conception,' etc. In the case of selecting a leader, some may give highest priority to those qualifications that will best help the group he leads to survive and grow; others may stress primarily his ability to accomplish aims that he values more highly than the group's survival.

In Fig. 1 we sketch an algorithm that identifies an optimal degree of precision in specifying needed knowledge. Let us take for example the group that feels as if it needs to know each candidate's managerial skill to remove some of the uncertainties. They enter their concern into an 'information system.' A good information system would not force its users to rephrase their concern with greater precision just so that it can be provided with information at the level of specificity it stores by asking as output such questions as, "will you accept performance score on test $X$ as an indicator of managerial skill?' It should inform the user that it cannot help him unless he specifies his concern with greater precision; it should help him to do this as well. Similarly, it should have some useful response at each level of specificity. If the user is too specific, he may not find any useful information at all; if he is too vague, he may get some useful information which lays buried in a vast array of items that are not useful, thus leaving him little better off than before consulting the information system. By adjusting a level-of-specificity knob in both directions, he may eventually reach a level where his request for knowledge is represented by "which reliable sources can give first hand accounts of this candidate's managerial skills'. Most employers do this right away and we argue that this contributes to the success of human systems beyond that which we have right or reason to expect.

Figure 2 provides an overview of our six attributes applied to task-situations of simple, moderate and high complexity. In each cell of the table we show a curve indicating how the probability of success in coping varies with the degree of precision in the representation of that aspect of the situation. These curves should be interpreted as hypotheses to be tested experimentally and derived from our model. It is clear from Fig. 2 that in more complex tasksituations more of our six attributes are characterized by an optimal degree of precision that falls between the extremes of 'most precise' and 'completely vague'. Here 'optimal' refers to maximization of the probability of coping.

As we face increasingly complex situations, our chances of coping are enhanced when we view these situations neither too vaguely nor too precisely, but at some appropriate level in between.

\section{CONCLUSION}

The precision with which we view a tasksituation is an important determinant of how 


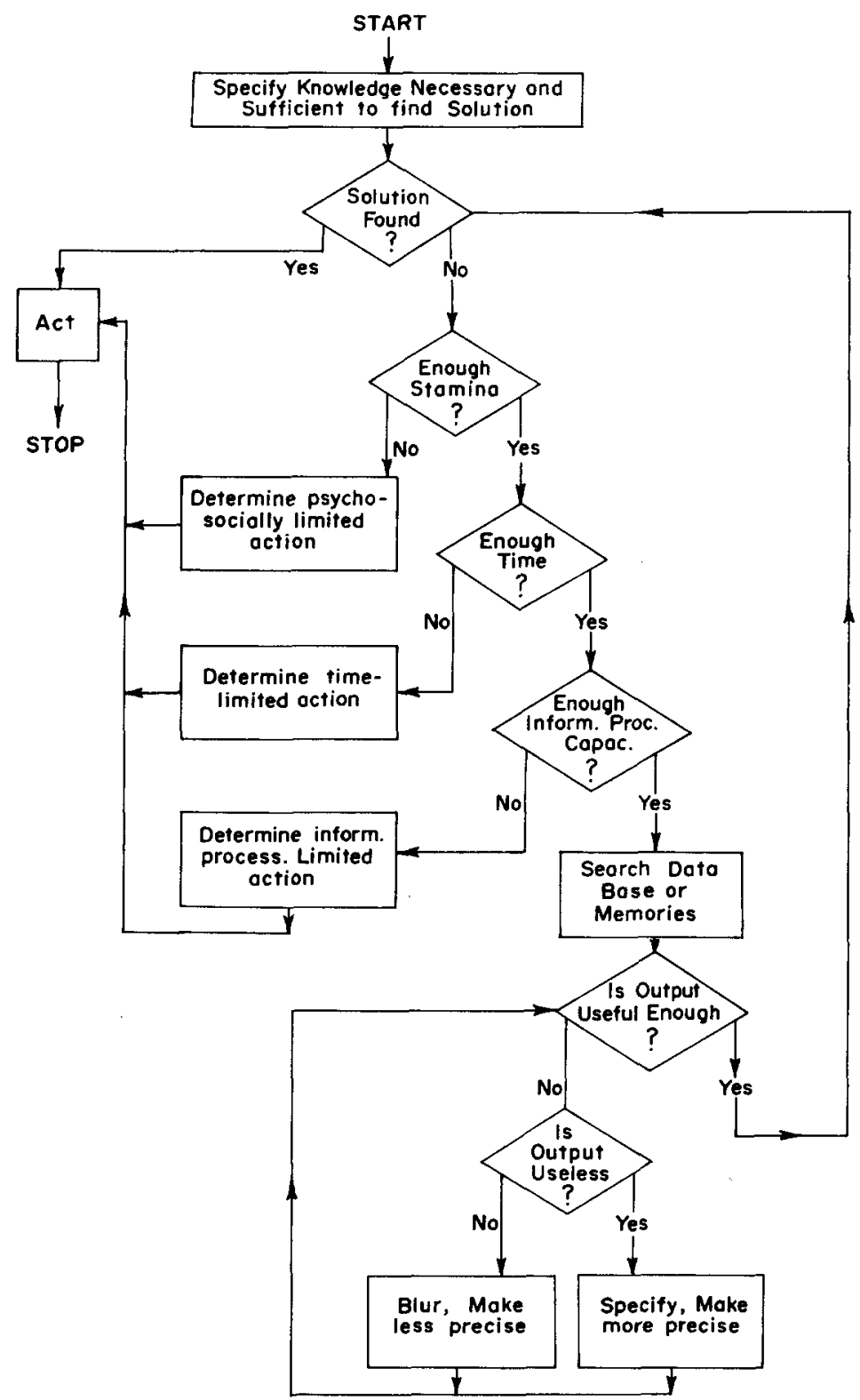

FIG. 1. Illustrating convergence to an optimal degree of precision in specifying knowledge needed to cope with a task situation. 


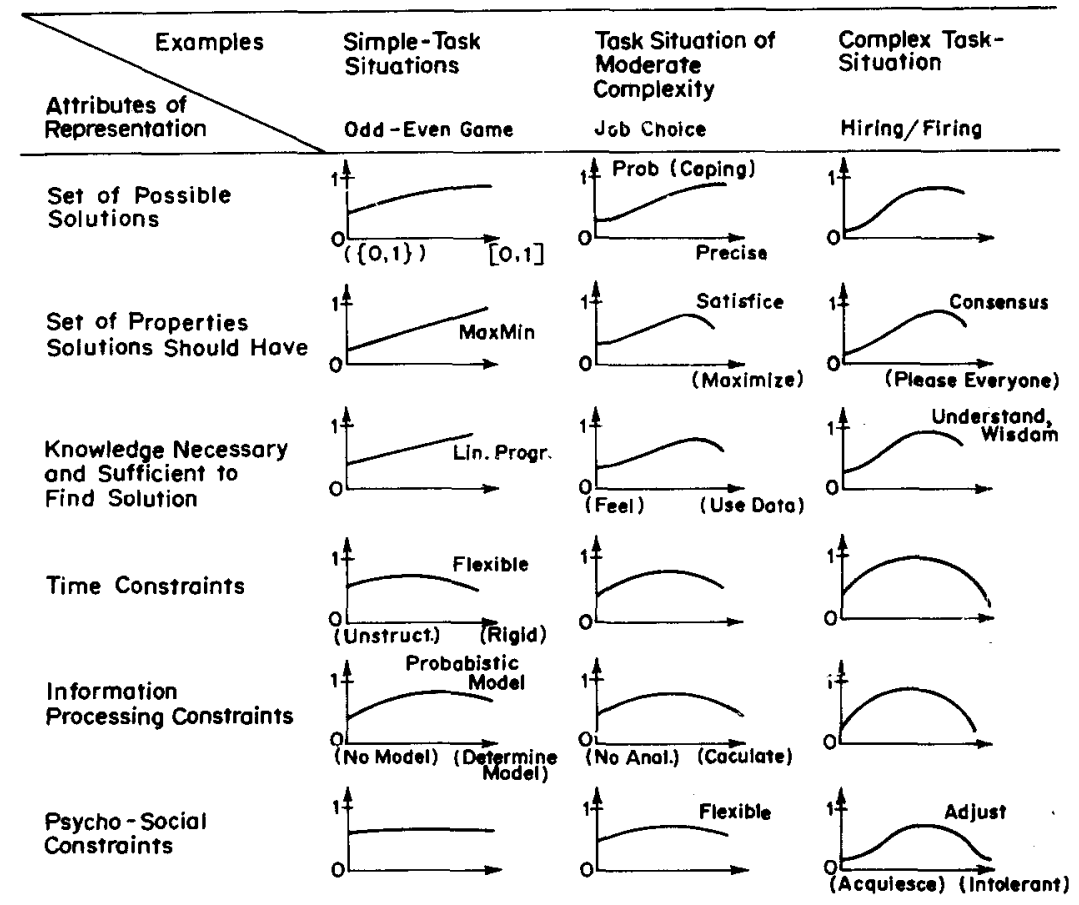

FIG. 2. Attributes of task-situations and their representations. ${ }^{1}$

well we cope with it. This assertion is based on a new qualitative model stimulated by several examples. It has yet to be tested by empirical methods. It corresponds to Mitroff's stress on the importance of 'global views of a problem,' but differs from the latter in stressing the importance of the exactitude with which such views are held. It is consonant with the findings of Tversky and Kahneman regarding the importance of frames of reference. It is premature to claim that the exact mathematical machinery of Fuzzy Set Theory is appropriate or necessary for the further development of our model.

\section{REFERENCES}

1. Amarfi S (1970) On the representation of problems and goal-directed procedures for computers. In Formal Systems and Non-numerical Problem Solving by Computer. (Eds BanerJI R \& Mesarovic M). Elsevier, USA.

2. AMAREL S(1970) Problem SOLVING AND DECISION MAKING

${ }^{1}$ The graph in each box indicates the form of the relation between probability of coping (ordinate) and the precision of the representation (abscissa), (e.g. Row 1, Col. 2).

To the right of each graph are keywords characterizing a good representation. Where it is useful to contrast this with a poor representation. such keywords are given in parentheses. by computer: an overview. In Cognition: a Multiple View (Ed. GaR VIN R). Spartan, New York, USA.

3. Chaltin GJ (1974) Information-theoretic limitations of formal systems. Il Ass. Comput. Mach. 21(3), 403.

4. Gallavotti G \& Ornstein D (1974) Billiards and Bernouilli schemes. Comm. Math. Phys. 38, 83-101.

5. Hunt EW \& Hovland C (1960) Computer simulation of concept attainment. Behav. Sci. 5(3), 265-267, 5C.

6. KOCHEN M (1955) Organized Systems with Discrete Information Transfer. PhD Thesis, Columbia University, New York, USA.

7. KoCHEN M (1960) Experimental study of hypothesisformation by computer. In Information on Theory (Ed. Cherry C), 377-403. Butterworth, London, UK.

8. KoCHEN M. (1960) Cognitive mechanisms. Internal report, RAP-3. IBM Research Center, Yorktown Heights, New York, USA.

9. KoHLeR W (1927) The Mentality of Apes. (Trans WiNTER E) 2nd ed. Harcourt Brace, New York, USA.

10. Minsky M (1961) Steps toward artificial intelligence. Proc. IEEE 49(1), 8-30.

11. Mintoff II (1977) Towards a theory of systemstic problem solving: prospects and paradoxes. Internat. $J l$ Gen. Syst. 4, 47-59.

12. Newell A \& Simon HA (1956) The logic theory machine: a complex information processing system. IEEE Trans. Info. Th. IT-2,3, 561-579 8A.

13. Platt J (1973) Social traps. Am. Psychol. 28(8), 641-651.

14. SCHUBERT LK (1974) Iterated limiting recursion and the program minimization problem. I/ Ass. Comput. Mach. 21(3), 436.

15. SimON HA (1955) Administrative Behavior. Macmillan, New York, USA

16. Snow CP (1960) The Masters. Scribner, New York, Hudson River ed., USA.

17. TVERSKY A \& KahNEMAN D (1974) Judgement under 
uncertainty: heuristics and biases. Science 185, ADDREss FOR CORRESPONDENCE: Manfred Kochen, Esq, 1124-1131.

18. Von Neumann J \& Morgenstern O (1944) Theory of Games and Economic Behaviour. 1st ed. Princeton University Press, USA.

19. WALD A (1950) Statistical Decision Functions. John Wiley, New York, USA.

Mental Health Research Institute, Department of Psychiatry, University of Michigan Medical Center, Ann Arbor, Michigan 48109, USA. 